\title{
Not Just a Laughing Matter: Disastrous Ending of Recreational Use of Laughing Gas
}

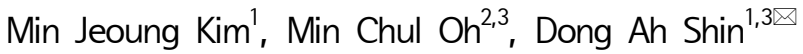 \\ ${ }^{1}$ Department of Neurosurgery, Yonsei University College of Medicine, Seoul, Republic of Korea \\ ${ }^{2}$ Department of Neurosurgery, Mediheal Neurosurgery Clinic, Seoul, Republic of Korea \\ ${ }^{3}$ Department of Neurosurgery, Yonsei Spine Research Institute, Yonsei University College of Medicine, Seoul, Republic of Korea
}

Nitrous oxide (N2O) is an established and widely used anesthetic gas that is also quite commonly misused for recreational purposes, due to its wide commercial availability. Overexposure can cause demyelination and eventual gliosis, within both the central and the peripheral nervous system. Here we present a patient with N2O-induced myelopathy following 3 months of recreational use. Despite its recognized neurotoxicity, the recreational abuse of $\mathrm{N} 2 \mathrm{O}$ is increasing and represents a major public health problem. The problem is however under-recognized and under-reported. We hope to issue a warning, raise public awareness, and initiate further research to prevent $\mathrm{N} 2 \mathrm{O}$ abuse.

Key Words: Nitrous oxide; Myelopathy; Public health

$\triangle$ Corresponding Author: Dong Ah Shin, Department of Neurosurgery, Yonsei Spine Research Institute, Yonsei University College of Medicine, 134 Shinchondong, Seodaemoon-Gu, Seoul 120-752, Republic of Korea Tel: +82-2-2228-2150, Fax: +82-02-393-9979, E-mail: cistern@yuhs.ac

\section{INTRODUCTION}

Nitrous oxide (N2O) gas traditionally used for surgical and den tal procedures is now available in grocery stores in the Republic of Korea. This gas is sometimes called "whippets" (after the containers it is sold in), "laughing gas", or the "epi- phany drug". Inhalation results in almost immediate psychot- ropic effects including euphoria, giggling, distortion of sound perception, and mild hallucinations. It has therefore been gaining popularity in recent years, especially among young people in nightclubs, and is considered a relatively innocuous substance. In fact, since Humphrey Davy reported the euphoric effects following self-administration of $\mathrm{N} 2 \mathrm{O}$ in $1799^{1)}$, it has become the $7^{\text {th }}$ most commonly used recreational drug worldwide and $8^{\text {th }}$ most commonly used substance in the UK, according to the Global Drug Survey 2016,3), and its recreational use in the Republic of Korea has similarly increased in recent years (Table 1). While most $\mathrm{N} 2 \mathrm{O}$ users are not aware of the harmful effects of this gas, multiple and repetitive inhalation is known to cause subacute degeneration of the spinal cord by inactivation of active vitamin B12, which leads to serious and fatal neurologic deficits $^{4,5)}$. Here, we report a case of a 25 -year-old man who developed myelopathy and peripheral neuropathy following 3 months of recreational $\mathrm{N} 2 \mathrm{O}$ use. We would like to raise awareness and emphasize the importance of proper management of such cases, to improve public health management, medically and socially.

\section{CASE REPORT}

Informed consent for publication of clinical data was obtained from the patient.

A 25-year-old man was admitted to our outpatient clinic, complaining of progressive quadriplegia for 3 days. He inhaled nitrous oxide gas all day for one month, at least 100 of balloon at each day at night club. There is no neurological deficit when he started to use the gas. He could walk only a few steps with assistance. Cranial nerve examination was normal. Muscle strength was reduced to grade 4 on the Medical Research Council Scale, and he showed areflexia, ataxic, and steppage gait. On

Table 1. Top ten substances used by UK

\begin{tabular}{l}
\hline Top ten substances \\
\hline 1. Alcohol \\
2. Tobacco \\
3. Cannabis \\
4. Ecstasy \\
5. Caffeinated energy drinks \\
6. Cocaine \\
7. Electronic cigarettes \\
8. Nitrous oxide \\
9. Ketamine \\
10. Codein
\end{tabular}


sensory examination, proprioception and his vibratory sense were remarkably intact, but the patient's light touch sensation, especially in the upper extremities, was impaired.

\section{Radiologic examination}

The patient's brain magnetic resonance imaging (MRI) findings were normal. Initial MRI of the whole spine showed high signal intensity on T2-weighted images in the dorsal columns of the cervical spine extending from $\mathrm{C} 2$ to $\mathrm{C} 6$, consistent with subacute degeneration of the spinal cord (Fig. 1), and suspicious thoracic arteriovenous malformations (AVM) in thoracic lesions. The initial diagnosis was cervical myelopathy due to N2O abuse or secondary to thoracic AVM. During laboratory and electromyographic examinations, the patient's lower leg weakness was found to have worsened. A follow-up cervical MRI showed more prominent and enlarged T2 signal intensity extending to $\mathrm{Cl}$ (Fig. 2). The patient's digital subtraction angiography (DSA) of the spinal cord was normal. The patient was treated with injections of $20 \mathrm{mg}$ dextrose (Intravenously administered, $10 \mathrm{mg}$ twice daily) and 1,000 $\mu \mathrm{g}$ /day vitamin B12.

\section{Laboratory examination}

Most laboratory tests were within the reference range, except for decreased serum levels of vitamin B12 $149 \mathrm{pg} / \mathrm{nL}$ (reference range: $250-900 \mathrm{pg} / \mathrm{nL}$ ). Serum folate and copper levels were normal. Serologic tests for syphilis, Epstein-Barr virus, cytomegalovirus, and human immunodeficiency virus antibodies were negative. The serum and urine homocyestein was not proceeded. Results of cerebrospinal fluid exams were also within the reference range (no $\mathrm{RBC}$, no $\mathrm{WBC}$, protein: $44.1 \mathrm{mg} / \mathrm{dL}$,

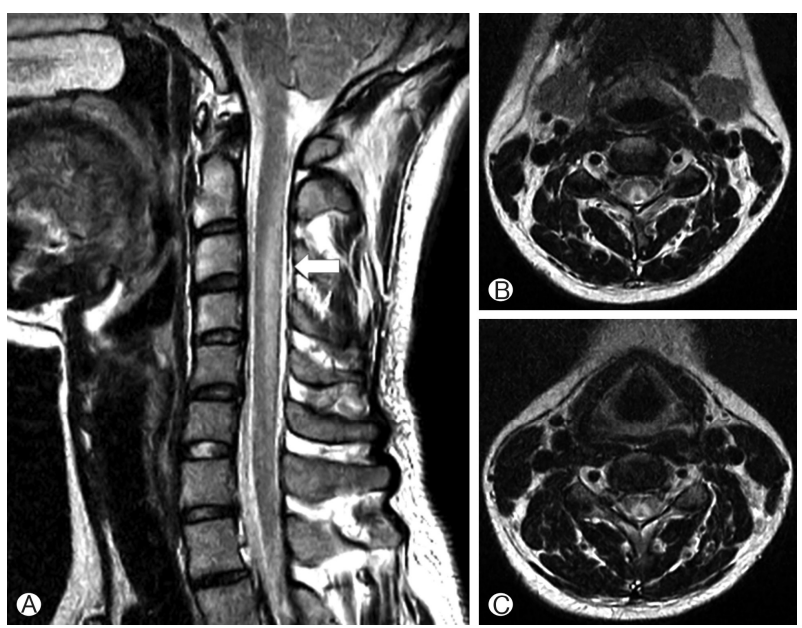

Fig. 1. T2-weighted sagittal image (A) and axial image (B), (C) showing increased signal intensity (arrow) in the cervical spinal cord (C2-C6). Axial image of C2-3(B). Axial image of C4-5(C) glucose: $57 \mathrm{mg} / \mathrm{dL})$.

\section{Electromyographic examination (EMG)}

Nerve conduction studies and electromyography showed a mild polyneuropathy with mixed axonal and demyelinating features.

Based on his history of $\mathrm{N} 2 \mathrm{O}$ inhalation, clinical features, and neuroimaging findings, a diagnosis of myelopathy secondary to $\mathrm{N} 2 \mathrm{O}$ intoxication was made. The patient was treated with injections of $1,000 \mu \mathrm{g} /$ day vitamin B12 daily for 7 days, followed by 1,000 $\mathrm{\mu g}$ orally weekly. One week later, although the tingling sensations and numbness in all extremities showed no improvement, his motor and lower extremities ataxia had rapidly improved.

\section{DISCUSSION}

$\mathrm{N} 2 \mathrm{O}$ has been used without any serious noticed or published side effects, and has been regarded innocuous, until Lassen and colleagues first reported on its toxicity in $1956^{6}$. This led to a thorough discussion on whether $\mathrm{N} 2 \mathrm{O}$ should still be used in anesthesia in the early 1980s.

$\mathrm{N} 2 \mathrm{O}$ toxicity can cause sensory predominant myeloneuropathy similar to subacute combined degeneration (SCD) caused by vitamin B12 deficiency. The related neuropathy is symmetrical and affects the legs more than the arms. It begins with pares thesia and ataxia associated with loss of vibration and position sense, and can progress to severe weakness, spasticity, clonus, paraplegia, and even fecal and urinary incontinence ${ }^{7-11)}$.

Many cases of clinical syndromes similar to our patient's initial presentation following recreational abuse of $\mathrm{N} 2 \mathrm{O}$ have been reported in the literature ${ }^{4,5,8,11)}$.

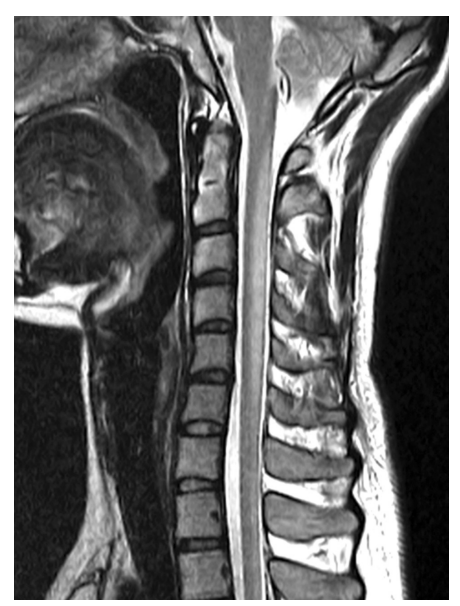

Fig. 2. T2-weighted sagittal image demonstrating more extensive cord signal changes from $\mathrm{C} 1$ to $\mathrm{C} 6$. 
As neurologic symptoms can occur prior to the development of the hematologic abnormalities of vitamin B1a2 deficiency, early recognition of the clinical symptoms and rapid initiation of treatment are essential to prevent progression and ameliorate clinical symptoms. Although most case series show promising outcomes after vitamin B12 replacement, significant improvements may be delayed and are often incomplete in patients with severe symptoms or those in whom treatment has been delayed $^{10,12,13)}$. In our case, vitamin B12 replacement was started 2 days after admission, because of delays due to laboratory and electromyographic examinations and a suspicious differential diagnosis of thoracic AVM. We performed follow-up MRI 2 days after the initial MRI scan, as the patient showed rapidly progressing weakness in his lower extremities, and found more extended high signal intensity on T2-weighted images. We therefore recommend starting vitamin B12 replacement as early as possible in cases of clinically suspected N2O-induced myelopathy. A thorough diagnostic evaluation utilizing diagnostic blood tests, electrodiagnostic examinations, and imaging is needed for correct diagnosis and proper management. While low serum cobalamin levels may be the only information needed to confirm the diagnosis, borderline or normal levels may occur. Normal vitamin B12 levels do not rule out the possibility of N2Oinduced SCD of the spinal cord, given that functional B12 deficiency can occur in the presence of normal serum vitamin B12 levels. In such patients, serum and urine homocysteine and methylmalonic acid metabolites can be used to confirm the diagnosis. MRI findings are characterized by edema secondary to demyelination and eventual gliosis resulting in increased $\mathrm{T} 2$ signals in the dorsal and, to a lesser extent, lateral and ante-

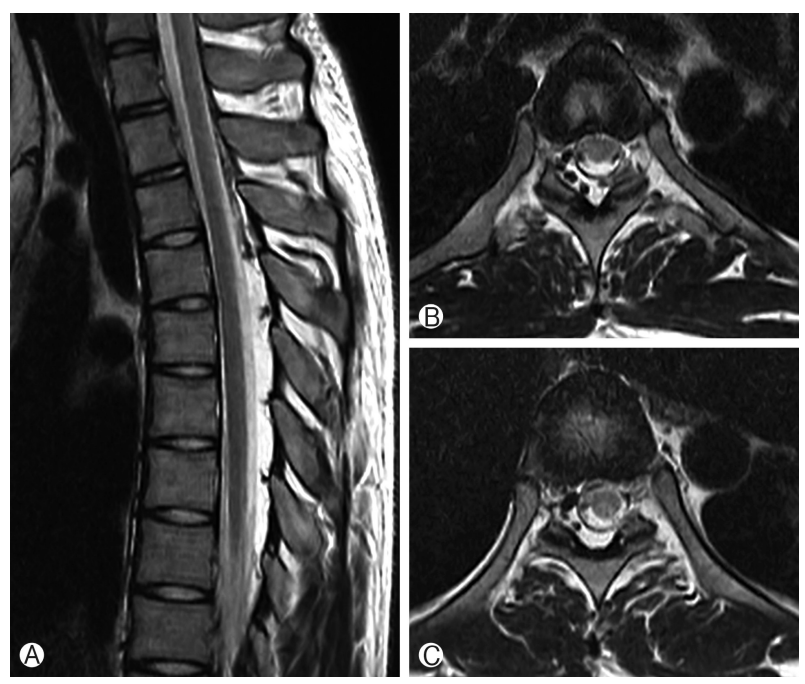

Fig. 3. T2-weighted sagittal (A) and axial image (B), (C)) showing engorged vascular lesions around thoracic cord, and diagnostic angiography with normal findings. rior columns of the spinal cord. A modest expansion of the spinal cord can be seen, and contrast enhancement is rare ${ }^{9,14,15)}$.

A differential diagnoses of abnormal signal lesions in the posterior columns of the spinal cord include transverse myelitis, lymphoma and other neoplasms, paraneoplastic myelopathy, multiple sclerosis, sarcoidosis, arterial or venous ischemia, and vascular malformations of the dura and spinal cord ${ }^{16,17)}$. In our case, initial MRI revealed suspicious thoracic vascular malformations (Fig. 3); however, diagnostic angiography findings were normal.

In summary, $\mathrm{N} 2 \mathrm{O}$ has recently gained popularity at parties in nightclubs and at music festivals in the Republic of Korea, due to its free availability, in the form of "whippets", aerosol canisters commonly used for whipped cream, each containing $8 \mathrm{~g}$ of $\mathrm{N} 2 \mathrm{O}$ (Fig. 1), and its low cost. Inhalant abuse of N2O is increasing in the Republic of Korea, similar to the United States and Europe, and clinical suspicions should be high, especially in young patients presenting with myelopathic symptoms of unclear etiology, since this represents a treatable and potentially reversible cause of myelopathy. We also think that public health systems should take a strong stance on restricting the drug's availability.

\section{REFERENCES}

1. Jay M. The atmosphere of heaven: The 1799 nitrous oxide researches reconsidered. Notes Rec R Soc Lond. Sep; 63(3):297-309, 2009

2. Global Drugs Survey, London, UK. http://www.globaldrugsurvey. com/wp-content/-themes/globaldrugsurvey/results/GDS2017_ key-findings-report_final.pdf. Accessed. 26 Feb; 2018.

3. Kaar SJ, Ferris J, Wāldron J, Devaney M, Ramsey J, Winstock AR. Up: The rise of nitrous oxide abuse. An international survey of contemporary nitrous oxide use. Journal of Psychopharmacology 30 (4):395-401, 2016

4. Sethi NK, Mullin P, Torgovnick J, Capasso G. Nitrous oxide "whippit" abuse presenting with cobalamin responsive psychosis. J Med Toxicol. Jun; 2(2):71-74, 2006

5. Thompson AG, Leite MI, Lunn MP, Bennett DL. Whippits, nitrous oxide and the dangers of legal highs. Pract Neurol Jun 15 (3):207-209, 2015

6. Lassen HC, Henriksen E, Neukirch F, Kristensen HS. Treatment of tetanus; Severe bone-marrow depression after prolonged nitrousoxide anaesthesia. Lancet. Apr; 270(6922):527-530, 1956

7. Alt RS, Morrissey RP, Gang MA, Hoffman RS, Schaumburg HH. Severe myeloneuropathy from acute high-dose nitrous oxide (N2O) abuse. J Emerg Med. Oct; 41(4):378-380, 2011

8. Cheng HM, Park JH, Hernstadt D. Subacute combined degeneration of the spinal cord following recreational nitrous oxide use. BMJ Case Rep. Mar; 8:2013, 2013

9. Karantanas AH, Markonis A, Bisbiyiannis G. Subacute combined degeneration of the spinal cord with involvement of the anterior columns: A new MRI finding. Neuroradiology. Feb 42(2):115117,2000 
10. Stabler SP. Vitamin B12 deficiency. N Engl J Med. May 368(21): 2041-2042, 2013

11. Waclawik AJ, Luzzio CC, Juhasz-Pocsine K, Hamilton V. Myeloneuropathy from nitrous oxide abuse: unusually high methylmalonic acid and homocysteine levels. WMJ 102(4):43-45, 2003

12. Goodman BP. Metabolic and toxic causes of myelopathy. Continuum (Minneap Minn). Feb; 21(1 Spinal Cord Disorders): 8499, 2015

13. Layzer RB. Myeloneuropathy after prolonged exposure to nitrous oxide. Lancet. Dec; 9 2(8102):1227-1230, 1978

14. Ilniczky S, Jelencsik I, Kenez J, Szirmai I. MR findings in subacute combined degeneration of the spinal cord caused by nitrous oxide anaesthesia--two cases. Eur J Neurol. Jan; 9(1):101-104, 2002

15. Ravina B, Loevner LA, Bank W. MR findings in subacute combined degeneration of the spinal cord: A case of reversible cervical myelopathy. AJR Am J Roentgenol. Mar; 174(3):863-865, 2000

16. Byun JS, Tsang ACO, Hilditch CA, et al. Presentation and outcomes of patients with thoracic and lumbosacral spinal epidural arteriovenous fistulas: A systematic review and meta-analysis. J Neurointerv Surg. Aug; 30, 2018.

17. Nasr DM, Brinjikji W, Clarke MJ, Lanzino G. Clinical presentation and treatment outcomes of spinal epidural arteriovenous fistulas. J Neurosurg Spine. May 26(5):613-620, 2017 